\title{
FABRICATION OF AN OCCLUSAL APPLIANCE USING A FULLY DIGITAL PROTOCOL: A TECHNICAL REPORT
}

\author{
IULIANA BABIUC ${ }^{1}$, LUCIAN CIUR ${ }^{2}$, MIHAELA PĂUNA ${ }^{3}$, MIHAI BURLIBAȘA ${ }^{4}$, GABRIELA \\ TĂNASE ${ }^{5}$, LILIANA BURLIBASA ${ }^{6}$, IRINA DONCIU ${ }^{7}$, IRINA ADRIANA BEURAN ${ }^{8}$, ILEANA \\ IONESCU ${ }^{9}$, CAMELIA IONESCU ${ }^{10}$
}

\author{
11,2,3,4,5,7,8,9,10 “Carol Davila” University of Medicine of Pharmacy Bucharest, ${ }^{6}$ University of Bucharest
}

\begin{abstract}
Keywords: $\quad$ occlusal Abstract: A good outcome of an occlusal appliance requires a well-adapted device, both in terms of appliance, digital occlusal relations and internal fit, which helps achieve patient comfort and compliance. Digital workflow, 3D printing, technology had led to the manufacturing of night guards that display a better accuracy and properties $C A D / C A M$ technology of the resin, ease of fabrication in terms of time and materials and the possibility of duplication. This technical report describes the clinical and laboratory steps that need to be followed to produce an occlusal appliance in a fully digital protocol. Intraoral scans were acquired, as well as digital occlusal records. A virtual $3 D$ dental device was designed using a CAD software, which was later manufactured via $3 D$ printing technology. The occlusal appliance displayed a good internal fit and enhanced properties of the resin. Minimal occlusal adjustments were required to secure functional guidance during protrusive and lateral movements of the mandible.
\end{abstract}

\section{INTRODUCTION}

Occlusal splints are commonly used for bruxism and temporomandibular disorders. Stabilization appliances, like the Michigan splint, have been shown to be an effective treatment of such cases.(1,2) A good outcome also requires a well-adjusted occlusal device, which facilitates patient comfort and compliance.(3) Predictable, optimal and consistent results have been reported when fabricating dental devices with the aid of digital technology.(4) The digital workflow consists in an initial intraoral optical impression, followed by the creation of a digital $3 \mathrm{D}$ object with a computer-assisted design (CAD) software and the transformation into a real object using computer-assistedmanufacturing (CAM) technology.

The accuracy of full arch intra-oral scan is considered to be within acceptable limits, but still challenging. Partial-arch digital impressions display a high degree of precision.(5) Also, the virtual occlusal records in full arch intraoral impressions are reported to be less predictable than in the case of quadrant scans. A tilting effect toward the side of the occlusal scan has been reported in full arch digital impressions, that reduces the sensitivity of the occlusal scan.(6) However, digital occlusal records have several advantages like no interposed medium between the teeth, ease of technique and ease of evaluation. In an analogue protocol, occlusal records have to be fitted in the laboratory and mounting stone casts and occlusal records in the articulator is also prone to significant errors.(7) From a clinical perspective, intraoral scan and a digital acquisition of the occlusal records help avoid the errors created by the materials and technique. A better internal fit has been reported, as well as reduced chair time for occlusal adjustment.(4)

Using CAD/CAM technology offers several technical advantages, like a better polymerization of the resin, lack of distortions and contractions, a reduced time for the production and adjustment of the device and the possibility to easily duplicate the appliance in case of fracture or wear.(8) In a fully
}

digital protocol, the occlusal devices can be milled or printed. New resins for 3D printers have been developed for occlusal devices, that are biocompatible, mechanically stable and wear resistant.(9-14)

\section{TECHNICAL REPORT}

1. A retractor for lips and cheek was placed, to keep them away from the teeth and help create a dry field. Suction was used to remove saliva from the dental surfaces and in order to reduce reflections.

2. A 3Shape Trios scanner was used. It was initially calibrated, both 3D and colour, to avoid distorsion

3. An intraoral scan is performed, using the technique recommended by the manufacturer. The lower arch is scanned first, with the mirror facing down (figure no. 1). Since in our case a lower appliance was needed, a good capture of all the dental areas, both occlusal and interdental, was required.

Figure no. 1. Intraoral digital impression of the lower arch. A detailed scan is recommended, capturing all the interdental spaces, for a good internal fit of the occlusal appliance

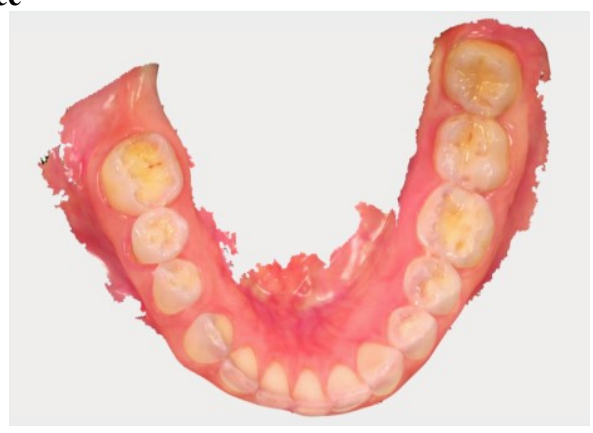

${ }^{4}$ Corresponding author: Mihai Burlibașa, Str. Plevnei, Nr. 19 Sector 1, Bucureşti, România, E-mail: mburlibasa@gmail.com, Phone: +407234 72632 Article received on 02.05.2020 and accepted for publication on 02.06.2020 


\section{CLINICAL ASPECTS}

4. The maxillary arch was scanned using the same technique, with the mirror facing up (figure no. 2)

Figure no. 2. Maxillary intraoral digital impression. The occlusal areas need to be scanned properly, in order to establish correct interarch contacts

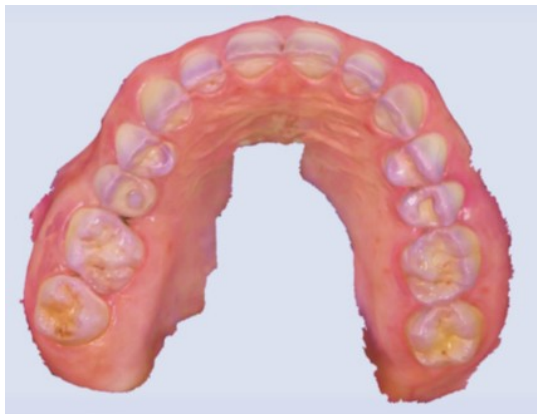

5. For occlusal records, the leaf-gauge was used (figure no. 3) Several maneuvers were performed in order to relax the lateral pterygoid muscles. This allows the elevator muscles to place the condyles in a comfortable, physiological position in the temporo-mandibular joints.

Figure no. 3. Establishing the intermaxillary position and space with the aid of leaf gauge

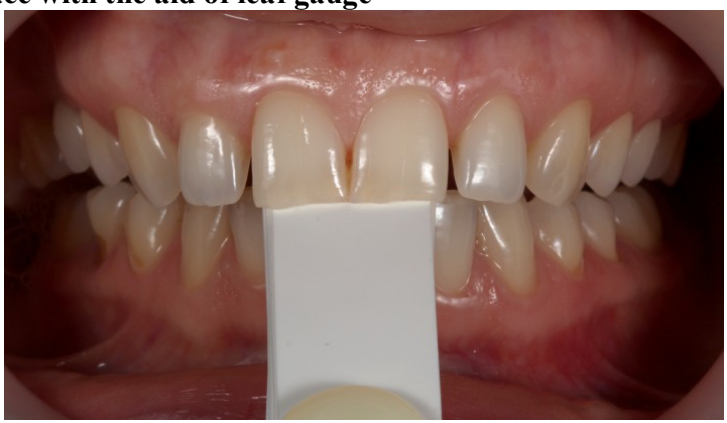

The interocclusal space was evaluated to make sure there is a sufficient gap between the arches to accommodate the occlusal splint (figure no. 4). An anterior obstacle also stabilizes the bite and prevents the tilting of the mandible during the digital registration of occlusion.

Figure no. 4. Digital impression of both arches with an established intermaxillary position and space

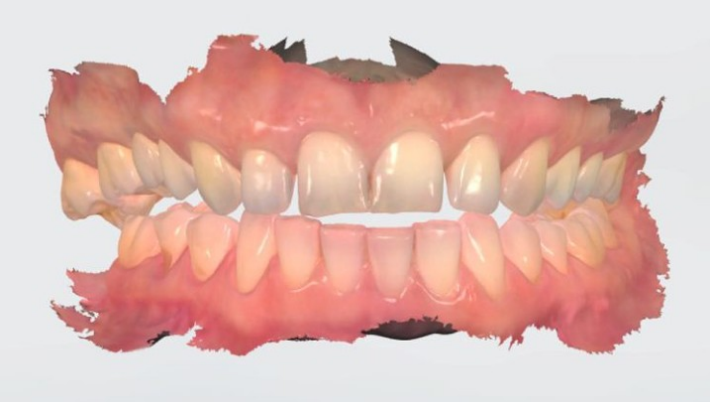

6. All the acquired data was sent to the dental laboratory in order to fabricate the occlusal appliance.

7. The digital impressions were imported in a CAD software (Exocad) in order to create a digital appliance. Several aspects were adjusted, like the amount of hard and soft tissue coverage and the distribution of occlusal contacts throughout the dental arch (figures no. 5, 6).

8. Once the design of the occlusal appliance was validated, it was printed using a resin designated for night guards and a 3D printer (figure no. 7).
Figure no. 5. Virtual occlusal appliance design using Exocad software

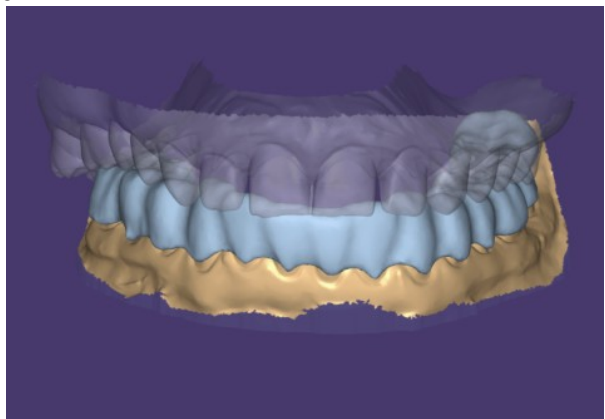

Figure no. 6. The distribution of the occlusal contacts on the virtual appliance, within the intermaxillary space and relation that was recorded clinically

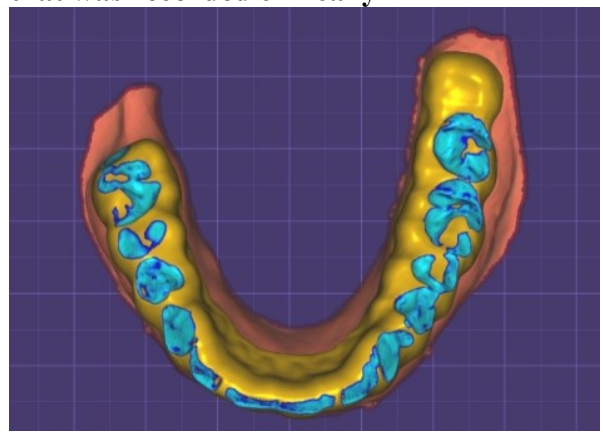

Figure no. 7. The occlusal appliance, manufactured with the aid of 3D printing technology

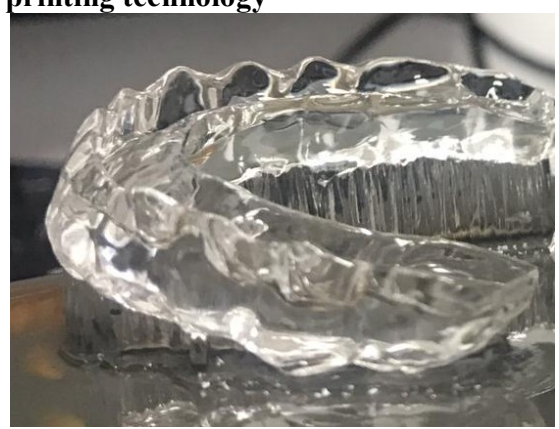

9. Two printed models were also produced. The dental office received the printed models of the two dental arches and the printed occlusal appliance (figures no. 8, 9).

Figure no. 8. Printed models of the clinical case, with an interposing occlusal appliance

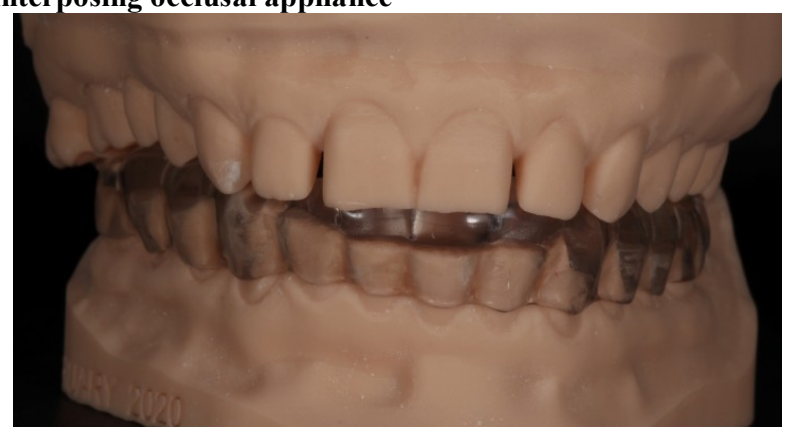

10. Minimal occlusal adjustments were necessary, especially in order to secure functional guidance of the mandible during protrusive and lateral movements. A good internal fit was observed, as well as a good quality of the printed resin. (figure no. 10) 
CLINICAL ASPECTS

Figure no. 9. Internal aspect of the occlusal appliance, fabricated with the aid of 3D printing technology

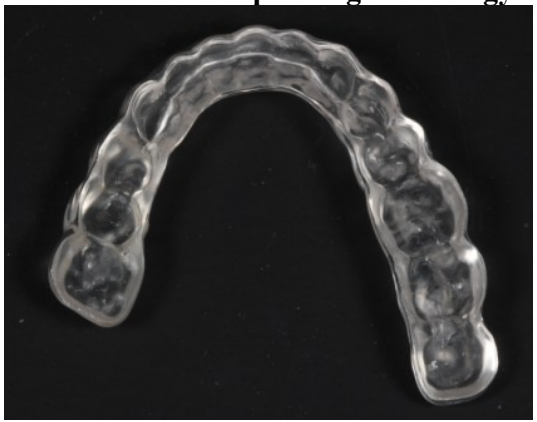

Figure no. 10. Clinical aspect of the occlusal appliance fabricated using a fully digital protocol

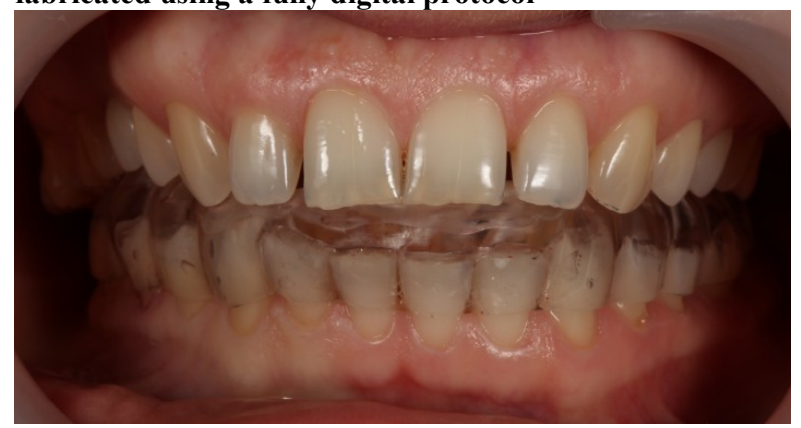

CONCLUSIONS

CAD/CAM technology allows for the creation of good quality occlusal appliances, with a reduced working time and an increased accuracy. 3D printing prevents the distorsions and contactions of the resin and allows for a better polymerization. In case of fracture and wear, these appliances can be easily duplicated.

Acknowledgement: In this article, all the authors have equal contribution with the first author.

\section{REFERENCES}

1. Al-Ani Z, Gray RJ, Davies SJ, Sloan P, Path FRC, Glanny AM. Stabilization splint therapy for the treatment of temporomandibular myofascial pain: A systematic review. J. Dent. Educ. 2005;69:1242-1250.

2. Proff P, Richter EJ, Blens T, Fanghanel J, Hutzen D, Kordab B, Gedrange T, Rottner K. A Michigan-type occlusal splint with spring-loades mandibular protrusion functionality for treatment of anterior disk dislocation with reduction. Ann of Anat. 2007;189:362-366

3. Friction J. Current evidence providing clarity in management of temporomandibular disorders: Summary of a systematic review of randomized clinical trials for intraoral appliances and occlusal therapies. J Evid Base Dent Pract. 2006;6:48-52.

4. Vasques M, Mori M, Lagana D. Three-dimensional printing of occlusal devices for temporomandibular disorders by using a free CAD software program: A technical report. J Prosthet Dent. 2020;123(2):232-235.

5. Ender A, Zimmermann M, Mehl A. Accuracy of completeand partial-arch impressions of actual intraoral scanning systems in vitro. Int J Computerized Dent. 2019;22(1):1119.

6. Edher F, Hannam A, Tobias D, Wyatt C. The accuracy of virtual interocclusal registration during intraoral scanning. J Prosthet Dent. 2018;120:904-912.

7. Radu M, Lazarescu F. Occlusion principles for the practicing dentist in the digital age. CAD/CAM.
2019;10(3):3-14

8. Lauren M, McIntyre F. A new computer assisted method for design and fabrication of occlusal splints. Am J Orthod Dentofacial Orthop. 2008;133:130-135.

9. Eftimie-Totu E, Cristache CM, Isildak S, Yildirim $\mathrm{R}$, Burlibașa M, Nigde M, Burlibașa L. Preliminary studies on citotoxicity and genotoxicity assesment of the PMMA $\mathrm{TiO}_{2}$ nanocompozites for stereolithographic complete dentures manufacturing. Revista de Chimie. 2018;69(5):1160-1165.

10. Bodnar DC, Burlibașa L, Vârlan C, Marcov N, Georgescu SR, Marcov CE. Mercury, biocompatibility and its impact on environment. Metalurgia International. 2009;14:95-100.

11. Burlibașa M, Tănase, Muntianu L, Murgu AI, Teodorescu E, Malița C. Quality of life, a multidisciplinary concept with economic and social impacts in medical practice. Metalurgia International. 2010; Vol. XV, Spec. Issue No. 4, p. $88-90$

12. Mocuța D, Popovici IA, Burlibașa L, Cristache G, Sfeatcu $\mathrm{R}$, Bodnar T. Impact of the living conditions on population health. Metalurgia International. 2009;14:17-19.

13. Ionescu CA, Popovici LR, Mocuța D, Malița C, Burlibașa M. The quality of human life from the perspective of sustainable development Metalurgia International. 2009;14:41-43.

14. Jamous S, Burlibaşa L. New insights in molecular analysis of gene regulation - an epigenetic overview. Letters in Applied Nanobioscience. 2018;(1-2):511-522. 